\title{
Research on Structural Adjustment and Countermeasures of the Development of Chinese Green Food Industry
}

\author{
Li Zhao \\ Practice Teaching Management Center, Harbin University of Commerce, Harbin, Heilongjiang Province, \\ China
}

Keywords: green food industry, structural constraints, transition of industry development mode, structure adjustment

\begin{abstract}
The development of green food industry is an industrial evolution process based on continuous optimization of structure. With the expansion of industrial scale, the overall development level of the industry in China has been continuously improved, the product structure has become more and more complete, the regional structure has been centralized, and the scale structure of enterprises has been continuously optimized. However, in the industrial development, the quality of industrial development is improved and the structure is optimized and adjusted. The development of green food industry is characterized by extensive features and the structural contradiction of industrial development is prominent. By analyzing the "structural constraints" in the development of China's green food industry, this paper proposes countermeasures and suggestions for transforming the industrial development mode and optimizing the industrial structure through the optimization of product structure, regional structure and enterprise scale structure.
\end{abstract}

\section{Introduction}

The green food industry is an emerging industry that has been developed and developed to meet the needs of agricultural industrial restructuring. It can effectively increase farmers' income and enhance the competitiveness of agricultural products market, and become a "win-win carrier" for coordinating economic development, ecological environment and people's health. After decades of rapid development of China's green food industry, industrial growth is mainly based on volume expansion. Industrial development is "big, fast" rather than "strong”, showing obvious extensive characteristics, still unable to adapt to national economic development and international There is still a big gap between the requirements of competition and the requirements of the scientific development concept and the new industrialization road. The fundamental reason for the persistence of this situation lies in the constraints of the development mode of traditional industries, and the lack of support for industrial development in the adjustment and adjustment of industrial structure. Therefore, the promotion of industrial development mode from extensive management to intensive management, from quantity growth to quality improvement is the only way to achieve sustainable development of green food industry. In the process of transforming the industrial development mode, the adjustment of industrial structure optimization is

The key is located.

\section{The status quo and characteristics of the development of green food industry}

The formation and development of China's green food industry is a typical government-driven type. In the early 1990s, China's agricultural development began to change from purely pursuing production to production, quality and efficiency. Driven by market demand, the Ministry of Agriculture pioneered the strategic decision to develop green food in 1990 and opened up the exploration process to promote the development of green food industry. 


\subsection{The scale of the industry continues to expand, the pace of development continues to accelerate, and the overall level of industrial development continues to increase.}

China's green food industry has now formed a relatively complete industrial system with a complete range of products, including base construction, product production and market circulation, with a certain scale and continuous improvement. From 1990 to 2015 the number of green food companies grew from 63 to9, 579, and the number of products increased from 127 to 23,386. In 2015, the environmental monitoring area of the production area was 2.6 billion mu, the annual sales of green food reached 4,383.2billion yuan, and export amount was 22.8 billion dollars.

\subsection{The product structure is becoming more and more complete, the regional structure is} centralized, and the enterprise scale structure is continuously optimized.

The product range is complete, and the proportion between primary products and processed products is gradually optimized. The green food products that have been developed include 57 subcategories of 4 categories, including agriculture, forestry, poultry, aquatic products and beverages, covering more than 1,000 varieties of agricultural products and processed foods.

The regional structure showed an obvious unbalanced development trend, and industrial agglomeration gradually appeared. The development and evolution of China's green food industry tends to be unbalanced and disordered. The industry is in a state of growth, and the industrial development and structure need to be adjusted and optimized. At the same time, the distribution of China's green food industry is uneven, showing a strong development trend of "concentration" and "distribution".

The construction speed of leading enterprises and large-scale production bases has accelerated, and the scale structure of enterprises has been gradually optimized. The green food industry has now gathered a large number of domestic agricultural products and food processing backbone enterprises.

\section{Analysis on the Structural Restriction of the Development of Green Food Industry}

China's green food industry has the basis and conditions for industrialization development in terms of total volume and development speed, but neglects the improvement of industrial development quality and structural optimization in industrial development, the extensive characteristics of industrial development, and the input and output of green food industry. The structural contradiction between the improvement of capacity and the rapid expansion of industrial scale is particularly prominent. Specifically in the following aspects:

\subsection{The relative development level of the industry is not high, and the structural contradiction of the products is prominent.}

From the perspective of the three industries of green food, the internal structure of the industry is not balanced, the advantages of primary agricultural and sideline products are outstanding, the green food processing industry and the catering industry are underdeveloped, and the industrial structure is dominated by low value-added products. In recent years, although the structure of green food industry has changed, it is still in a low-level stage in the industrial upgrading sequence. The green food primary industry still has an absolute advantage. The primary products account for a large proportion, and the proportion of refined processing products is small. Judging from the large-scale structure of green food products, the structure and types of green food products in China are single. Among them, the proportion of agriculture, forestry and processed products is relatively large and the drought is gradually rising; followed by livestock processing and beverage products with a relatively small proportion, while the proportion of aquatic products with large market demand is small and rising. Not obvious. 


\subsection{The distribution of the green food industry is uneven. The regional distribution of China's green food industry development shows a strong development trend of "concentration" and “distribution".}

Among them, Jiangsu, Shandong, Heilongjiang, Liaoning, Inner Mongolia and other top ten provinces have more than $60 \%$ of the total number of green foods. The regional advantages of Anhui, Jilin, Sichuan, Fijian, Zhejiang, Hubei and other provinces are gradually emerging. The distribution is more fragmented. The regional distribution structure of China's green food industry development is dynamically changing and tending to production. The production and operation are scattered, lack of unified planning, and the cluster development is lagging behind. The spatial optimization of industrial resources has not been realized.

\subsection{The scale structure of enterprises is dominated by small and medium-sized enterprises,} and the advantages of industrial organization have not been fully utilized.

China has a large number of green food enterprises and a small scale of production. Enterprises cannot obtain scale effects and enhance their ability to resist risks. The degree of industrial organization and integration is low, and there is a lack of leading enterprise groups. The advantages of industrialization are not obvious, and the brand management ability is weak. The development of China's green food industry has the characteristics of "high-speed and low-efficiency" expansion of quantity, and the scale of green food production bases and enterprises is small, which directly affects the leading enterprises' ability to influence the development of industrial clusters and ultimately affects the competitiveness improvement of green food industry.

\section{Suggestions on the adjustment of green food industry structure}

In the process of rapid development of green food industry, the industrial development mode has obvious characteristics. The promotion of industrial structure has not been fully reflected in the promotion of industrial development. "Structural constraints" have become the impact of the improvement of the efficiency of green food industry and the coordinated development of industry. The prominent factor. Without the transformation of industrial development mode and the optimization and adjustment of industrial structure, there will be no major leap and improvement in the development of green food industry.

The optimization and adjustment of the green food industry structure should be based on the optimization and adjustment of product structure, relying on the green food advantage industrial zone to support the industrial base and supporting the leading enterprises in processing and circulation, and realize the "technology-driven product support, regional planning and industrial organization". To play a role in promoting the industrial development of the "aggregation effect" and "scale effect" of the green food industry. Specifically, it includes technical structure, product structure, regional structure, scale structure, etc.:

\subsection{The optimization and upgrading of technical structure is the core of the optimization and upgrading of industrial structure.}

It is necessary to pay attention to the role of technology in the optimization and upgrading of industrial structure and industrial development. Through technological structure optimization and upgrading to improve the variety structure and improve product quality, enhance the independent innovation capability of the industry and enhance the stamina of industrial development. The promotion of government industrial policies is an important guarantee and main driving force for technological innovation and technological progress, and plays a role in supporting, guiding and "amplifying" the government's industrial policies for technological R\&D and promotion.

Through the formulation and implementation of technical policies, relying on scientific and technological progress and innovation, we will increase investment in green food technology development and production. Innovative green food product research and development model, with new products, new technologies, new technologies as a link, encourage and support universities and 
research institutes to carry out green food cooperative research, push government departments and institutions of higher learning, research institutes and related industries The joint technology research and development model strengthens the cultivation of advantages and high-end products by focusing on supporting the development of green food-leading products and deep processing of products.

\subsection{The optimization and adjustment of green food product structure should follow the law of industrial evolution. Actively promote the diversification of product structure.}

Relying on high value-added and deep processing products, and optimize the upgrading of product structure to promote the development of the second and third industries of green food and the optimization and upgrading of industrial structure. Product structure optimization and adjustment should be based on the existing advantageous product sequence, improve the orderly and balanced structure of the green food industry, promote the realization of economies of scale and product quality, and achieve the brand effect and industrialization of leading product development.

\subsection{The optimization of regional structure of green food industry should give full play to the basic role of market allocation resources.}

The government should strengthen the macro guidance for the development of green food industry. Actively adjust the layout of the green food industry, adhere to the principle of comparative advantage and highlighting key points, implement the unbalanced development strategy, and comprehensively consider the spatial distribution differences of factors such as the supply conditions of resources (resources) and production conditions of the green food industry. Large, local conditions, support and support, give priority to the development of green food advantageous industry type areas with certain foundation and development potential, support and rely on the dominant industry type areas to create a strong green food industry and core competitiveness. The green food industry continues to advantage Regional concentration and the regional advantages of the green food industry gradually emerged, achieving industrial agglomeration and relative concentration, exerting its role in radiating, guiding and pulling the regional economy, and ultimately promoting the "balanced" development of the green food industry regional layout.

\section{Acknowledgment}

This research was supported by Philosophy and Social Sciences Planning Project in Heilongjiang (No.16JYB14); Humanities and Social Sciences Planning Project of Heilongjiang Education Department (No.12542060); and Ph.D. research project of Harbin Universe of Commerce (No. 14RW14).

\section{References}

[1] John Adams, Wang Jialiang. Industrial clusters and regional economic development in China: the case of "green" food [J].Journal of Chinese Entrepreneurship, 2009, 1(3): 279-294.

[2] Export specialization and economic nrowth around the world [J]. Economic systems, 2011(35): 45-63

[3] Wang Dezhang, Chen Jiangbo, Du Huiyong. Changes in Regional Competitiveness of China's Green Food Industry: Current Situation, Problems and Countermeasures[J].Journal of Daqing Normal University,2012(4): 37-42

[4] Song Guoyu, Zhao Li. Intrinsic Mechanism and Empirical Study on the Transformation of Green Food Industry Development Mode Based on C-D Production Function[J].Agriculture Economics and Management,2012(1): 28-34

[5] Zhao Dawei. Study on the Development Stage of Green Food Industry and the Transformation of Growth Mode [J].Academic Exchange.2013 (2):55-58 